\title{
Low intrinsic emittance in modern photoinjector brightness
}

\author{
Christopher M. Pierce $\odot$, Matthew B. Andorf $\odot$, Edmond Lu, Colwyn Gulliford $\odot$, \\ Ivan V. Bazarov, and Jared M. Maxson \\ Cornell Laboratory for Accelerator-based Sciences and Education, Cornell University, \\ Ithaca, New York 14853, USA \\ Matthew Gordon $\odot$ and Young-Kee Kim® \\ University of Chicago, Chicago, Illinois 60637, USA
}

Nora P. Norvell, Bruce M. Dunham®, and Tor O. Raubenheimer®

SLAC National Accelerator Laboratory, Menlo Park, California 94025, USA

(Received 16 April 2020; accepted 16 June 2020; published 21 July 2020)

\begin{abstract}
Reducing the intrinsic emittance of photocathodes is one of the most promising routes to improving the brightness of electron sources. However, when emittance growth occurs during beam transport (for example, due to space charge), it is possible that this emittance growth overwhelms the contribution of the photocathode, and, thus, in this case source emittance improvements are not beneficial. Using multiobjective genetic optimization, we investigate the role intrinsic emittance plays in determining the final emittance of several space-charge-dominated photoinjectors, including those for high-repetition-rate free electron lasers and ultrafast electron diffraction. We introduce a new metric to predict the scale of photocathode emittance improvements that remain beneficial and explain how additional tuning is required to take full advantage of new photocathode technologies. Additionally, we determine the scale of emittance growth due to point-to-point Coulomb interactions with a fast tree-based space-charge solver. Our results show that, in the realistic high-brightness photoinjector applications under study, the reduction of thermal emittance to values as low as $50 \mathrm{pm} / \mu \mathrm{m}(1 \mathrm{meV}$ mean transverse energy) remains a viable option for the improvement of beam brightness.
\end{abstract}

DOI: 10.1103/PhysRevAccelBeams.23.070101

\section{INTRODUCTION}

Improving the brightness of space-charge-dominated electron sources will unlock a wealth of next-generation accelerator physics applications. For example, the largest unit cell that may be studied with single-shot ultrafast electron diffraction (UED) is limited by the beam's transverse coherence length, which is determined by transverse emittance, at a high enough bunch charge to mitigate the effects of shot noise in data collection. The study of protein dynamics with UED requires producing $>1 \mathrm{~nm}$ scale coherence lengths at more than $10^{5}$ electrons and subpicosecond pulse lengths at the sample location [1,2]. In another example, the intensity of coherent radiation available to the users of free electron lasers (FELs) is, in part, limited by beam brightness. Beam brightness affects the efficiency,

\footnotetext{
*cmp285@cornell.edu
}

Published by the American Physical Society under the terms of the Creative Commons Attribution 4.0 International license. Further distribution of this work must maintain attribution to the author(s) and the published article's title, journal citation, and DOI. radiated power, gain length, and photon energy reach of FELs $[3,4]$.

Photoinjectors equipped with low intrinsic emittance photocathodes are among the brightest electron sources in use today. Peak brightness at the source is limited by two factors: the electric field at the cathode and the photocathode's transverse momentum spread. Several shortpulse Child-Langmuir-like charge density limits have been derived for the photoemission regimes of relevance to practical photoinjectors. These current density extraction limits make explicit the dependence of peak brightness on photocathode parameters and the electric field [5-7]. Depending on the aspect ratio of the bunch, the brightness limit is superlinear in the electric field and motivates the push toward high accelerating gradient photoinjectors. Contemporary $\mathrm{dc}$, normal-conducting rf (NCRF), and superconducting rf (SRF) photoelectron guns have peak accelerating fields of the order of $10 \mathrm{MV} / \mathrm{m}$ [8-11] with very high repetition rates (well above $1 \mathrm{MHz}$ ). At the cost of duty factor, state of the art NCRF electron guns can offer even higher fields of the order of $100 \mathrm{MV} / \mathrm{m}$ [12], and recent experimental results suggest the possibility of pushing peak fields to nearly $500 \mathrm{MV} / \mathrm{m}$ for cryogenically cooled accelerating structures [13-24]. 
In this work, we characterize the intrinsic emittance at the photocathode source via the mean transverse energy (MTE):

$$
\varepsilon_{\mathrm{C}}=\sigma_{x} \sqrt{\frac{\mathrm{MTE}}{m c^{2}}},
$$

where $\sigma_{x}$ is the laser spot size and $m$ is the mass of the electron. Here, it is clear that MTE plays the role of an effective temperature of emission.

Great progress is being made in the discovery of lowMTE photocathodes which are expected to improve the usable brightness of photoinjectors. Because of the practical trade-offs involved with photocathode choice, most photoinjectors today use materials with an MTE of around $150 \mathrm{meV}$ [25-28]. At the cost of quantum efficiency (QE), this MTE may be reduced by tuning the driving laser's wavelength. For example, in $\mathrm{Cs}_{3} \mathrm{Sb}$ and Cs:GaAs photocathodes, the lowest MTE that may be achieved via wavelength tuning at room temperature is nearly 35 and $25 \mathrm{meV}$, respectively, but at $10^{-6}-10^{-5} \mathrm{QE}$ [29-31]. Recent work has shown that the cryogenic cooling of photocathodes emitting at threshold can reduce MTE even further, potentially down to single-digit meV MTEs [32]. However, a natural question arises amid this progress in MTE reduction: In modern space-charge-dominated applications, to what extent does MTE reduction actually improve the final emittance?

Even in the case of linear transport, 3D space-charge effects lead to a transverse position-angle correlation which varies along the longitudinal coordinate and leads to an inflation of projected emittance that requires compensation [33-36]. The residual emittance after compensation is due to nonlinear forces, from either space-charge or beam line elements. Scaling laws exist to help estimate their effects $[37,38]$. In some cases, nonlinearity can cause phase space wave breaking in unevenly distributed beams that is a source of irreversible emittance growth $[39,40]$. Another irreversible cause of emittance growth is disorder-induced heating (DIH) and other Coulomb scattering effects which are expected to become important in the cold dense beams of future accelerator applications [41]. Avoiding these emittance growth mechanisms requires the advanced design and tuning of photoinjector systems.

Multiobjective genetic algorithm (MOGA) optimization is a popular technique for the design and tuning of realistic photoinjectors [42-48]. Photoinjectors often have to balance several key design parameters or objectives that determine the usefulness of the system for a given application. MOGA is a derivative-free method for computing the Pareto front, or family of highest performing solutions, in a parallel and sample efficient manner [49]. Elitist genetic algorithms are known to converge to the global optima of sufficiently well-behaved fitness functions given enough evaluations [50]. This makes them well suited for problems involving many local extrema. Practical problems often require optimizations to be performed over a constrained search space, and there exist techniques of incorporating these constraints into existing genetic algorithms without sacrificing efficiency [51].

In this work, we examine the limits beam transport places on the ability of photoinjectors to take advantage of low-MTE photocathodes in a diverse set of realistic simulated photoinjectors that have been tuned by a MOGA for ultimate performance. This article begins with a discussion of our results involving the simulations of beam lines with idealized zero-emittance photocathodes. These simulations are performed on three important examples of high-brightness electron beam applications: highrepetition-rate FELs, as well as single-shot dc and rf-based UED devices. Using zero cathode emittance simulations, we introduce a new metric called the characteristic MTE to help understand the scale of photocathode MTE which is relevant to final beam quality. It is shown that, depending on the properties of the beam line, system parameters need to be reoptimized to take full advantage of photocathode improvements. We present a method of estimating when reoptimization needs to be performed and the magnitude of its effect on final emittance. Finally, we set the scale for the magnitude of emittance growth due to point-to-point Coulomb interactions using a stochastic space-charge algorithm.

\section{OPTIMIZATIONS WITH A 0 meV MTE PHOTOCATHODE}

To understand the contribution of photocathode MTE toward the final emittance of high-brightness photoinjectors, we directly compare injector performance with a contemporary $\sim 150 \mathrm{meV}$ MTE photocathode to what would be achievable with a perfect $0 \mathrm{meV}$ MTE counterpart. To cover the wide range of existing and near-future accelerator technologies, we chose three realistic beam lines with significantly different energies as a representative set of high-brightness photoinjector applications. A dc and NCRF electron-gun-based single-shot UED beam line reflect the two predominant energy scales of electron diffraction with single nanometer-scale emittance at 10-100 fC bunch charge: order of magnitude $100 \mathrm{keV}$ and $1 \mathrm{MeV}$, respectively. At higher bunch charge, we select an SRF photoinjector under development at Japan's High Energy Accelerator Research Organization (KEK) expected to be capable of submicrometer-scale emittance at $100 \mathrm{pC}$ bunch charge for simulations representative of FEL driver applications. We consider these beam lines to be space-charge dominated based off of their expected applications, which are anticipated to push their machine settings into a range where space-charge forces become important.

The ultimate performance of each system is evaluated on the basis of the particle-tracking codes General Particle 
(a) dc Gun UED
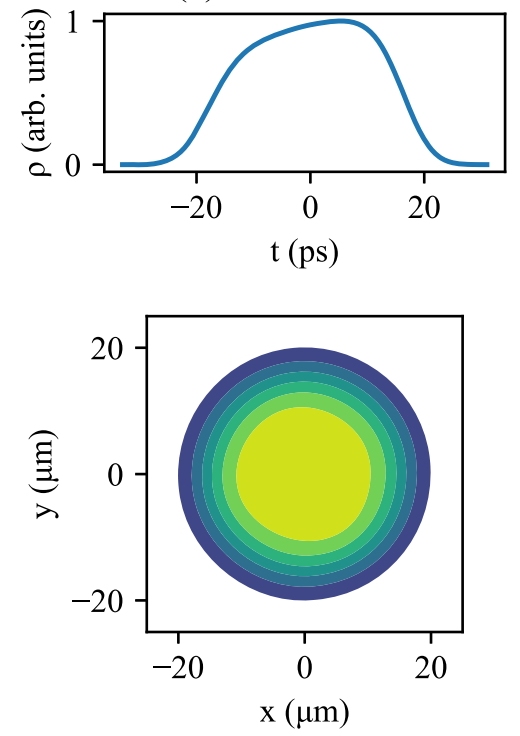

(b) NCRF Gun UED
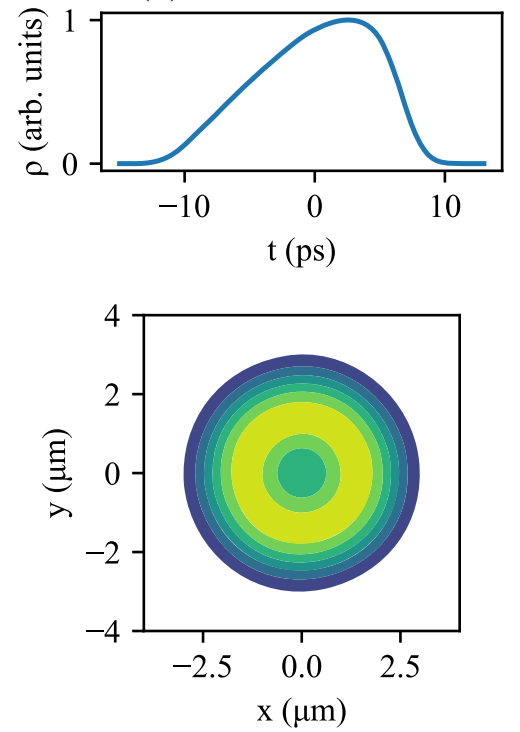

(c) KEK Gun FEL Driver
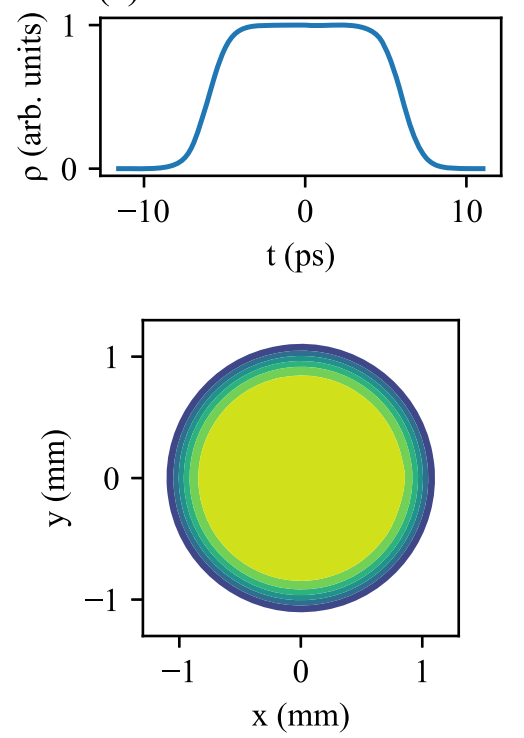

FIG. 1. An example of the initial longitudinal and transverse spatial distributions of the beam for each system. These examples were selected from the $0 \mathrm{meV}$ Pareto front and are the same individuals plotted in Fig. 5.

Tracer (GPT) [52] and ASTRA [53] with optimization carried out in the framework of MOGA. Particle-tracking simulations have been shown to have good correspondence with the dynamics of real beam lines [54]. Children were generated with simulated binary crossover and polynomial mutation [55]. Selection was performed with strength pareto evolutionary algorithm II (SPEA-II) [56] in the case of both UED examples and with non-dominated sorting genetic algorithm II (NSGA-II) [49] in the case of the FEL example. Emittance preservation is known to depend strongly on the initial transverse and longitudinal distribution of the beam. To this end, the optimizer is given the power to change parameters controlling the initial particle distribution. In particular, the optimizer may change the size and length of the beam while preserving cylindrical symmetry. The shape of the beam along the radial and (a) dc Gun UED

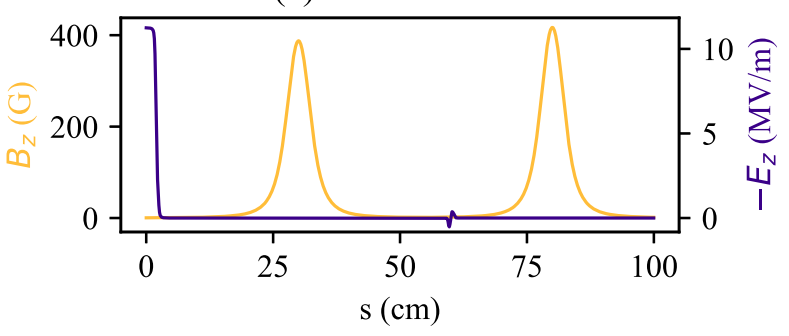

(b) NCRF Gun UED

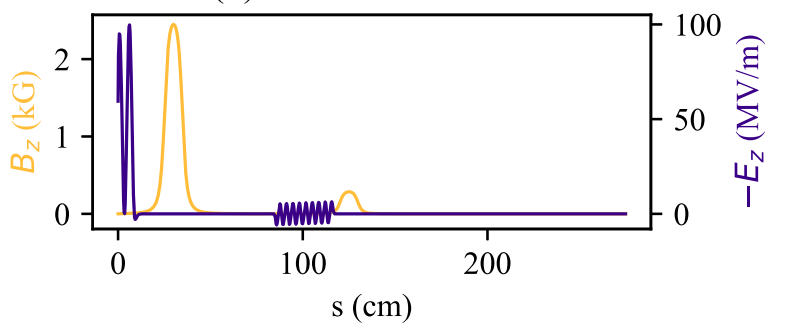

(c) KEK Gun FEL Driver

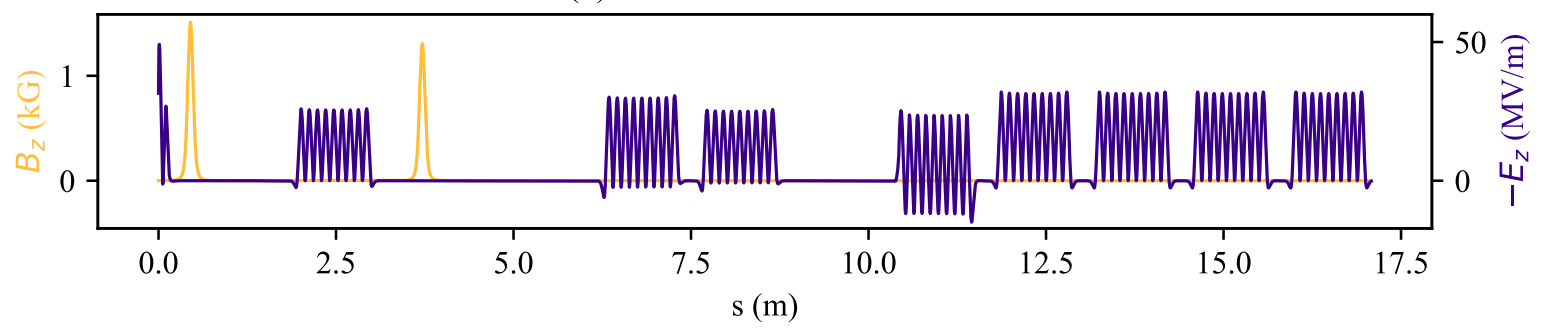

FIG. 2. The on-axis electric and magnetic field as seen by a reference particle in the center of the electron bunch. In each subfigure, the cavity and magnet parameters are taken from an individual along the $0 \mathrm{meV}$ Pareto front of the respective beam line. Fields are output directly from General Particle Tracer. In the case of the FEL, fields are computed from the energy change $(\mathrm{d} E / \mathrm{d} s)$ and Larmor angle output from ASTRA as a function of the position. 
TABLE I. Optimizer configuration for the de gun UED beam line.

\begin{tabular}{lc}
\hline \hline Decision & Range \\
\hline Bunch charge & $0-160 \mathrm{fC}$ \\
Initial rms beam size & $0-1 \mathrm{~mm}$ \\
Initial rms beam length & $0-50 \mathrm{ps}$ \\
MTE & $0,150 \mathrm{meV}$ \\
Gun voltage & $225 \mathrm{kV}$ \\
Solenoid current 1 and 2 & $0-4 \mathrm{~A}$ \\
Buncher voltage & $0-60 \mathrm{kV}$ \\
Buncher phase & $90^{\circ}$ \\
\hline \hline Objective & Goal \\
\hline rms emittance & Minimize \\
Delivered bunch charge & Maximize \\
\hline \hline Constraint & Value \\
\hline Final rms spot size & $<100 \mu \mathrm{m}$ \\
Final rms bunch length & $<1 \mathrm{ps}$ \\
\hline \hline
\end{tabular}

longitudinal axes is interpolated between the set of four distributions described in Ref. [38]. Example initial distributions taken from one individual for each beam line are shown in Fig. 1.

The dc UED beam line is modeled after a similar system under development at Cornell University using the cryogenically cooled photoemission source described in Ref. [57]. The performance of this system under different conditions than presently considered is discussed in

TABLE II. Optimizer configuration for the NCRF UED beam line.

\begin{tabular}{lc}
\hline \hline Decision & Range \\
\hline Bunch charge & $0-300 \mathrm{fC}$ \\
Initial rms beam size & $0-50 \mu \mathrm{m}$ \\
Initial rms beam length & $0-50 \mathrm{ps}$ \\
MTE & $0,150 \mathrm{meV}$ \\
Gun phase & $-90^{\circ}-90^{\circ}$ \\
Peak gun field & $100 \mathrm{MV} / \mathrm{m}$ \\
Beam energy & $4.5 \mathrm{MeV}$ \\
Solenoid current 1 and 2 & $0-4 \mathrm{~A}$ \\
Buncher peak power & $0-25 \mathrm{MW}$ \\
Buncher phase & $90^{\circ}$ \\
\hline \hline Objective & Goal \\
\hline rms emittance & Minimize \\
Delivered bunch charge & Maximize \\
\hline \hline Constraint & Value \\
\hline Final rms spot size & $<100 \mu \mathrm{m}$ \\
Final rms bunch length & $<1 \mathrm{ps}$ \\
\hline \hline
\end{tabular}

Ref. [58], where a detailed description of the layout and simulation methodology is also provided. On-axis fields for this beam line are shown in Fig. 2(a). The beam line consists of two solenoids that surround an NCRF singlecell bunching cavity and aid in transporting the highbrightness beam to the sample located at $s=1 \mathrm{~m}$. The optimizer is given control over all magnet and cavity settings to minimize the rms emittance at the sample while maximizing bunch charge. Only solutions that keep the final spot size smaller than $100 \mu \mathrm{m} \mathrm{rms}$ and the final beam length less than $1 \mathrm{ps}$ rms are considered. These constraints were chosen based on common sample sizes used in diffraction [25] and the timescale of lattice vibration dynamics [59,60]. For a complete description of the decisions, objectives, and constraints used for this system, refer to Table I.

The high-gradient NCRF UED beam line is driven by a 1.6-cell $2.856 \mathrm{GHz}$ gun capable of $100 \mathrm{MV} / \mathrm{m}$ and based on a design currently in use at a number of labs [25,61-64]. Samples are located at $s=2.75 \mathrm{~m}$, and the optimizer is given full control over two solenoids which surround a nine-cell bunching cavity that is modeled after the first cell of the SLAC linac described in Ref. [65]. A discussion of

TABLE III. Optimizer configuration for the KEK gun FEL driver example.

\begin{tabular}{lc}
\hline \hline Decision & Range \\
\hline Bunch charge & $100 \mathrm{pC}$ \\
Initial rms beam size & $0.05-10 \mathrm{~mm}$ \\
Initial rms beam length & $5-70 \mathrm{ps}$ \\
MTE & $0,130 \mathrm{meV}$ \\
Gun gradient & $20-50 \mathrm{MV} / \mathrm{m}$ \\
Gun phase & $-60^{\circ}-60^{\circ}$ \\
Gun energy & $1.5-3.5 \mathrm{MeV}$ \\
Solenoid 1 field & $0-0.4 \mathrm{~T}$ \\
Capture cavity gradient & $0-32 \mathrm{MV} / \mathrm{m}$ \\
Capture cavity phase & $-180^{\circ}-180^{\circ}$ \\
Capture cavity offset & $0-2 \mathrm{~m}$ \\
Solenoid 2 field & $0-0.3 \mathrm{~T}$ \\
Solenoid 2 offset & $0-2 \mathrm{~m}$ \\
Cryomodule offset & $0-3 \mathrm{~m}$ \\
Accel. cavity 1, 2, and 4 field & $0-32 \mathrm{MV} / \mathrm{m}$ \\
Accel. cavity 1, 2, and 4 phase & $-90^{\circ}-90^{\circ}$ \\
\hline \hline Objective & $\mathrm{Goal}$ \\
\hline rms emittance & $\mathrm{Minimize}$ \\
Final rms bunch length & $\mathrm{Minimize}$ \\
\hline \hline Constraint & $<90 \mathrm{MeV}$ \\
\hline Final energy & $<200 \mathrm{keV}$ \\
Energy spread & $<5 \mathrm{keV}$ \\
\hline Higher-order energy spread & \\
\hline \hline &
\end{tabular}

${ }^{\mathrm{a}}$ Gun energy is computed from gradient and phase and not directly controlled by optimizer. 
our previous optimization experience with this beam line under a different set of constraints can be found in Ref. [66]. As in the case of the dc UED beam line, the optimizer was configured to minimize final rms emittance while maximizing delivered bunch charge under the constraint of keeping the final spot size less than $100 \mu \mathrm{m}$ rms and the final length shorter than $1 \mathrm{ps}$ rms. The decisions, objectives, and constraints of this optimization are detailed in Table II, and an example of the on-axis fields from an optimized individual is shown in Fig. 2(b).

Our FEL driver example includes a 1.5 -cell $1.3 \mathrm{GHz}$ SRF gun in development at KEK for use in a CW energy recovery linac (ERL) light source coupled with a photoinjector lattice aimed at use in the linac coherent light source II high energy upgrade (LCLS-II HE) [67]. The gun energy is controlled by the optimizer but is in the range $1.5-3.5 \mathrm{MeV}$. Immediately after the gun is a $1.3 \mathrm{GHz}$ nine-cell capture cavity surrounded by two solenoids. The remaining cavities, of the same design as the capture cavity, are shown in the plot of external fields in Fig. 2(c) and accelerate the beam to its final energy of roughly $100 \mathrm{MeV}$. Accelerating cavity number three was kept off during optimization as a planned backup for cavity failure in the real machine. The bunch charge was fixed to $100 \mathrm{pC}$, and optimizations were performed to minimize both rms emittance and bunch length at the end of the injector

(a) dc Gun UED
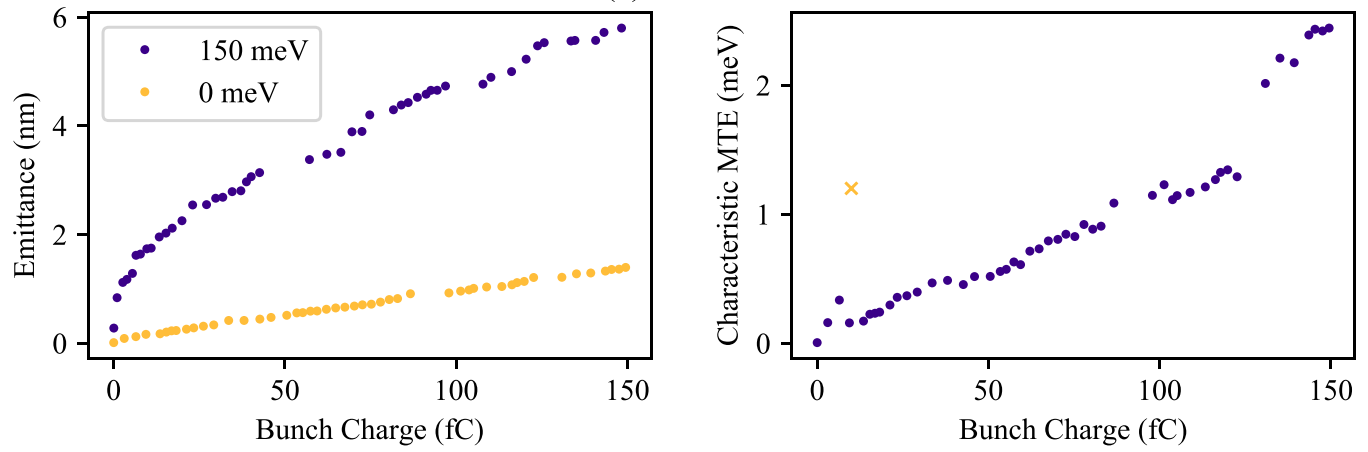

(b) NCRF Gun UED


(c) KEK Gun FEL Driver
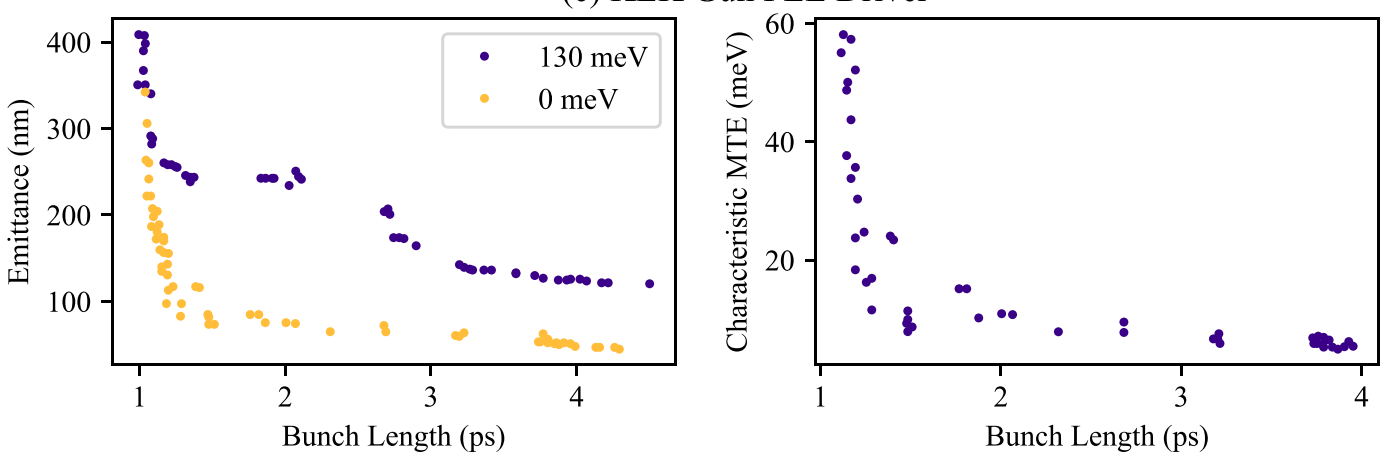

FIG. 3. The Pareto fronts of each beam line for the $\sim 150$ and $0 \mathrm{meV}$ MTE photocathodes and their characteristic MTE. The UED examples show between a factor of 10 and 100 improvement in brightness between the two Pareto fronts. The characteristic MTE calculated from a simulation including the effects of Coulomb scattering is included for the dc and NCRF gun UED examples as a yellow cross. 




FIG. 4. The probability distribution of initial spot sizes among the optimized individuals. The three example beam lines are labeled by color, and individuals from the $\sim 150 \mathrm{meV}$ fronts are in dashed lines, while the individuals from the $0 \mathrm{meV}$ fronts are represented by solid lines.

system. Energy constraints were tailored for the injector's use in the LCLS-II HE upgrade, and so we required valid solutions to have an energy greater than $90 \mathrm{MeV}$, an energy spread below $200 \mathrm{keV}$, and a higher-order energy spread less than $5 \mathrm{keV}$. The full set of decisions, objectives, and constraints is compiled in Table III.

Initial generations of the genetic optimization were evaluated with a small number of macroparticles to develop a good approximation of the global optima before moving on to the more accurate simulations involving $10^{5}$ macroparticles for the UED examples and $10^{4}$ macroparticles for the FEL driver. The optimization stopping condition was that improvement of the Pareto front with each successive generation fell below a threshold of approximately $10 \%$ relative change. The products of these optimizations are shown in Fig. 3.

Both UED beam lines show a factor of between 10 and 100 improvement in brightness when the $150 \mathrm{meV}$ photocathode is replaced by its $0 \mathrm{meV}$ counterpart. The degree of improvement is slightly greater in the case of the dc gun UED beam line. As seen in Fig. 4, the optimizer chooses a smaller initial spot size for the NCRF gun individuals than for the dc gun individuals. We conjecture that this is enabled by the higher accelerating gradient of the NCRF gun limiting the effects of space-charge emittance growth. Because of the fact that initial emittance depends on both the photocathode's MTE and the initial spot size, a smaller initial spot size can mitigate the effects of a high thermal emittance photocathode. The NCRF beam line also outperforms the dc beam line for emittance in absolute terms at similar bunch charges, further suggesting a benefit with higher gradients on the cathode. There is a sharp rise in slice emittance while the beam is still inside the gun and at low energy seen in Figs. 5(a) and 5(b). This suggests that nonlinear space-charge forces play a role in the residual emittance, and the higher gradient and energy of the NCRF example could explain why it outperforms the dc example. We observed that the brightness improvement from the $0 \mathrm{meV}$ photocathode was limited to a factor of 10 in the case of the FEL driver. The higher bunch charge of this application is expected to increase the role of space-charge forces in transport and could be a cause of this more modest improvement.

At the end of every beam line and for each individual, we calculated the parameter

$$
\rho=\frac{I}{I_{0}} \cdot \frac{\sigma_{x}^{2}}{\beta \gamma \varepsilon^{2}},
$$

where $I_{0}$ is the Alfvén current, $I$ is the average current, and $\beta$ and $\gamma$ have their typical definitions in special relativity. This represents the ratio of the space-charge and emittance pressure terms in the beam envelope equation. It was greater than one in all cases, which confirms that all of the beam lines operating with optimized settings are spacecharge dominated. The ratio $\rho$ was larger by at least an order of magnitude for individuals from the $0 \mathrm{meV}$ Pareto fronts than for individuals from the 150 and $130 \mathrm{meV}$ fronts for comparable bunch charge or length.

\section{THE CHARACTERISTIC MTE METRIC}

As long as the beam dynamics of the system do not change significantly with the introduction of a new photocathode, we can use the heuristic relationship that nonzero initial emittance will add roughly in quadrature with the emittance due to beam transport, and the final emittance will be

$$
\varepsilon^{2} \approx \varepsilon_{\mathrm{T}}^{2}+\sigma_{x, i}^{2} \frac{\mathrm{MTE}}{m c^{2}},
$$

where $\varepsilon_{\mathrm{T}}$ is the emittance gained in beam transport, $\sigma_{x, i}$ is the initial spot size, and $\varepsilon_{\mathrm{C}}=\sigma_{x, i} \sqrt{\frac{\mathrm{MTE}}{m c^{2}}}$ is the initial emittance due to the photocathode and initial spot size. To understand when the photocathode's MTE is important in the final emittance, we define a characteristic MTE that would result in the emittance contribution of the photocathode and beam transport being equal as

$$
\operatorname{MTE}_{C}=m c^{2}\left(\frac{\varepsilon_{\mathrm{T}}}{\sigma_{x, i}}\right)^{2} .
$$

The characteristic MTE is a beam-line-specific quantity that sets the scale for when photocathodes play a significant role in determining the final emittance of a photoinjector. Photocathode improvements down to the characteristic 
(a) dc Gun UED
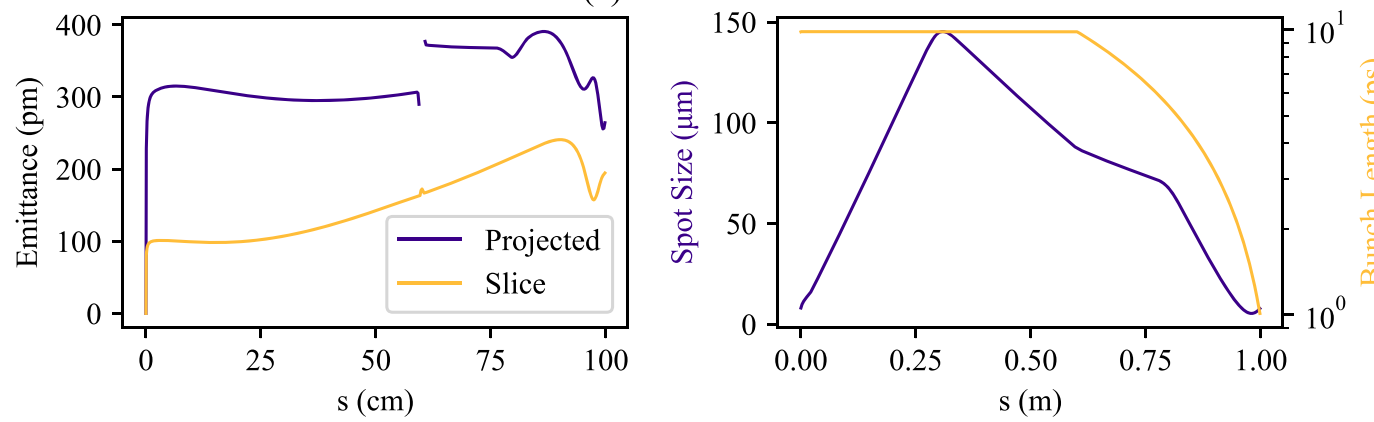

(b) NCRF Gun UED
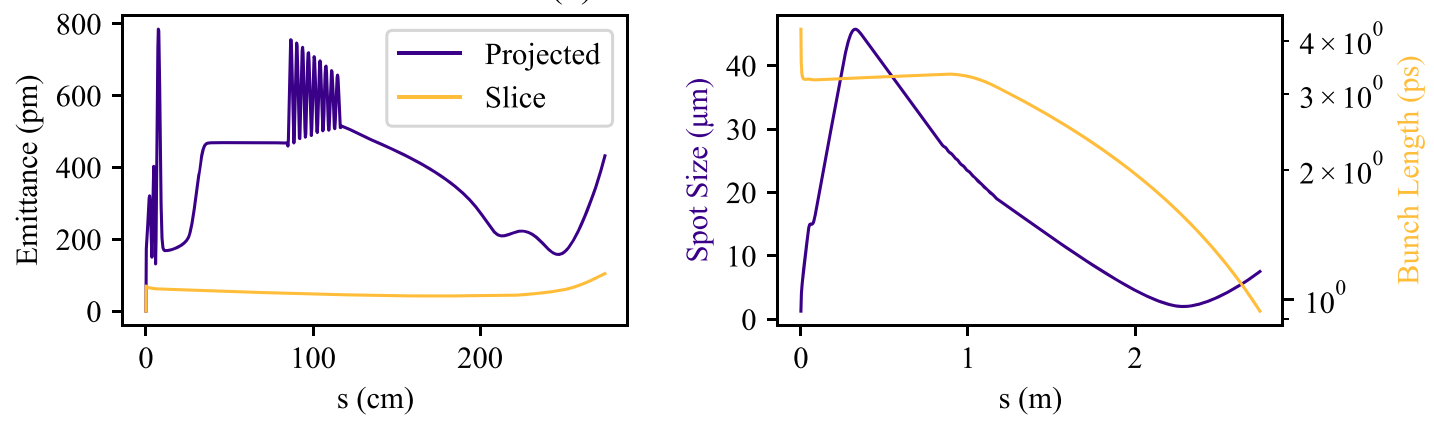

(c) KEK Gun FEL Driver
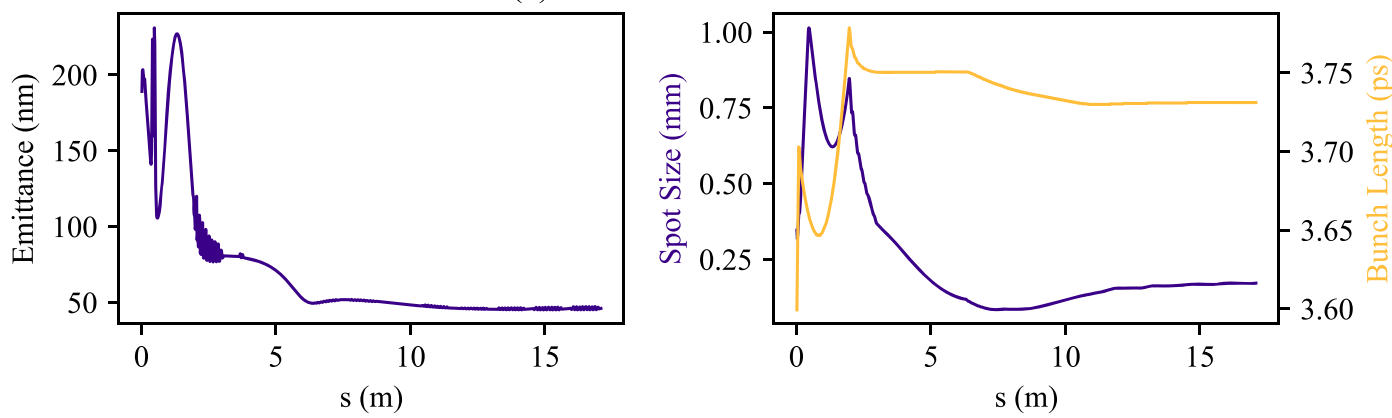

FIG. 5. Emittance and beam sizes for an individual along the $0 \mathrm{meV}$ Pareto front of each example. The UED individuals were selected at $16 \mathrm{fC}$ bunch charge. The projected emittance is the typical rms normalized transverse emittance, and the slice emittance is the average of the emittance evaluated over 100 longitudinal slices. The beam width and length are also plotted for reference. The total projected emittance in (a) is clipped at 500 pm for clarity.

MTE are likely to translate into increased usable brightness.

The characteristic MTE of each example is shown in Fig. 3. Photocathode improvements down to the level of single-meV MTE do affect the final emittance of each photoinjector application studied here. The characteristic MTE of the NCRF UED and FEL driver examples increases to roughly 15 and $50 \mathrm{meV}$ at high bunch charge and short bunch length, respectively. The larger characteristic MTE of the NCRF UED example is likely due to the smaller initial spot size of the individuals. This can be seen in Fig. 4. That smaller spot size will increase the characteristic MTE for the same emittance, because the initial emittance is less sensitive to photocathode parameters. Characteristic MTEs at short bunch lengths in the FEL example are primarily limited by large emittance growth in beam transport.

To test the validity of the heuristic argument that initial and transport emittance should add in quadrature, we simulated each individual from the $0 \mathrm{meV}$ Pareto fronts with a photocathode whose MTE is the characteristic MTE. The final emittance is expected to grow by a factor of $\sqrt{2}$, and we observe the ratio to be close to but slightly larger than that value. The frequency of ratios for each beam line is plotted in Fig. 6. For our investigation, we assume that the insertion of a new photocathode does not significantly change beam transport. However, this condition will be violated to some extent and could explain why the ratio observed is slightly larger than $\sqrt{2}$. 


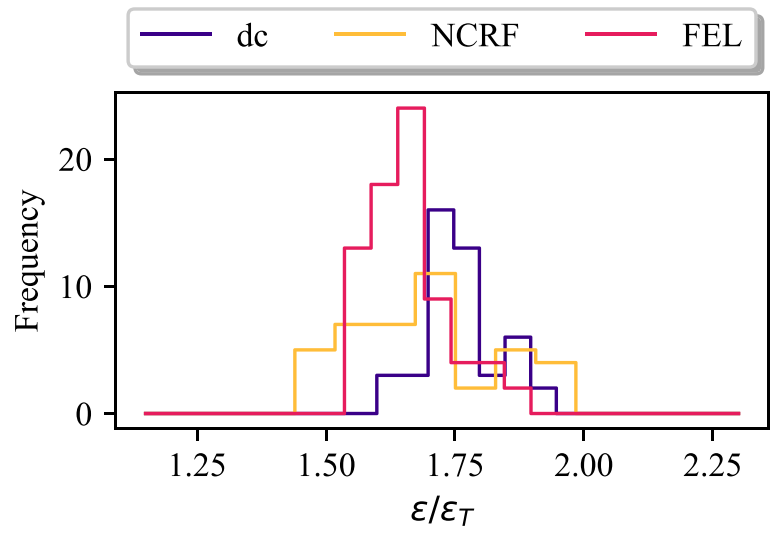

FIG. 6. Individuals from the $0 \mathrm{meV}$ beam line were resimulated with a photocathode MTE equal to their characteristic MTE. The probability distribution of the ratio of the new final emittance to the original final emittance is plotted.

\section{REOPTIMIZATION FOR NEW PHOTOCATHODES}

Our optimization experience showed that taking full advantage of the initial emittance improvements afforded by a new low-MTE photocathode required the reoptimization of beam line parameters. In particular, when individuals from the $150 \mathrm{meV}$ Pareto fronts of the UED beam lines are resimulated with a $0 \mathrm{meV}$ photocathode and no changes to beam line parameters, their emittance is more than 50\% larger than the emittance of individuals in the $0 \mathrm{meV}$ Pareto front at comparable bunch charge. This can be understood by considering the sensitivity of the transport emittance optimum to small changes in the initial spot size.

The characteristic MTE analysis does not take into account the fact that if shrinking the initial spot size from its optimal value reduces the initial emittance more than it increases emittance growth in transport, then the overall emittance will still go down. The initial emittance, as in Eq. (1), can be reduced by using a smaller initial spot size. However, if the system was already at the initial spot size which minimizes emittance growth in transport, as is the case of individuals along the $0 \mathrm{meV}$ Pareto front, then changing it will negatively affect beam line performance. Since the final emittance is roughly the quadrature sum of the initial emittance and the growth during transport, there will be a trade-off in minimizing both the initial emittance and emittance growth. If the system was previously optimized with a high-MTE photocathode, then the optimal spot size will not be at the minimum transport emittance possible, and new low-MTE photocathodes can unlock strategies the optimizer avoided due to their larger spot sizes which increase initial emittance. In this case, reoptimization will be required upon the insertion of a new low-MTE photocathode.

This trade-off is represented graphically in Fig. 7 by plotting emittance as a function of the initial spot size.
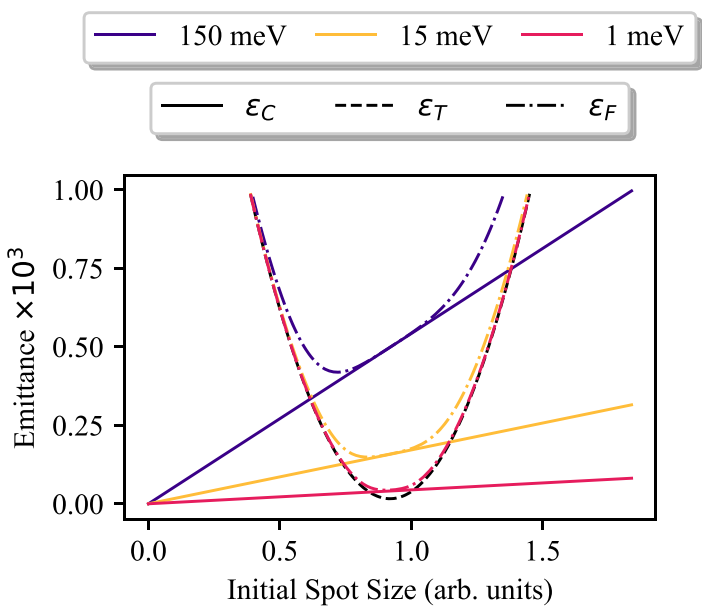

FIG. 7. An illustration of how reoptimization may be required upon insertion of a new photocathode. In black is the emittance due to transport $\left(\varepsilon_{\mathrm{T}}\right)$ as a function of the initial spot size. Around the optimal spot size $\sigma_{x, i, 0}$, this is approximately quadratic. The sensitivity in this example is roughly $x \approx 0.001$. The solid lines represent the initial emittance $\left(\varepsilon_{\mathrm{C}}\right)$ for three different thermal emittances. The dashed lines are the final emittance $\left(\varepsilon_{\mathrm{F}}\right)$ or the quadrature sums of initial and transport emittance. The optimal spot size with the $150 \mathrm{meV}$ photocathode is significantly smaller than with a 0 or even $1 \mathrm{meV}$ photocathode.

Initial emittance is linear in the initial spot size and is represented by a line whose slope depends on photocathode MTE. Close to the optimum, the emittance due to transport may be expressed as a polynomial expansion in $\sigma_{x, i}$ which, to lowest order, is quadratic. The final emittance is roughly the quadrature sum of both terms and has an optima at a smaller spot size than for transport emittance alone. The characteristic MTE can also be represented in this plot, since the initial emittance for a photocathode with an MTE equal to the characteristic MTE will pass through the vertex of the transport emittance parabola.

By using the second-order expansion of beam transport's contribution to the emittance $\left(\varepsilon_{\mathrm{T}}\right)$ as a function of initial spot size $\left(\sigma_{x, i}\right)$ around the optimum,

$$
\varepsilon_{\mathrm{T}}\left(\sigma_{x, i}\right)=A\left(\sigma_{x, i}-\sigma_{x, i, 0}\right)^{2}+\varepsilon_{\mathrm{T}, 0},
$$

we can find the new optimal emittance with nonzero MTE. Here $\sigma_{x, i, 0}$ is the optimal spot size, and $\varepsilon_{\mathrm{T}, 0}$ is the optimal emittance. To simplify our discussion, we consider the case of optima that are highly sensitive to changes in the initial spot size. Define the unitless parameter $x=\varepsilon_{\mathrm{T}, 0} /\left(A \sigma_{x, i, 0}^{2}\right)$ to measure the optimum's sensitivity. In the limit of sensitive optima $(x \ll 1)$, the new smallest emittance when the initial spot size is allowed to vary is

$$
\varepsilon_{\mathrm{opt}}^{2}=\varepsilon_{\mathrm{T}, 0}^{2}+\varepsilon_{\mathrm{C}}^{2}\left[1-\frac{x}{2} \frac{\mathrm{MTE}}{\mathrm{MTE}_{C}}\right](x \ll 1) .
$$


The new optimal initial spot size will be smaller for the nonzero MTE photocathode and, in the limit of small $x$, is approximately

$$
\sigma_{x, i, \mathrm{opt}}^{2}=\sigma_{x, i, 0}^{2}\left[1-x \frac{\mathrm{MTE}}{\mathrm{MTE}_{C}}\right] \quad(x \ll 1) .
$$

In practice, we observe the tendency of the optimizer to choose smaller initial spot sizes for beam lines with nonzero photocathode MTE. In Fig. 4, we plot the frequency of initial spot sizes from the 0 and $\sim 150 \mathrm{meV}$ Pareto fronts of each beam line. For the UED examples, the initial spot sizes for individuals in the $150 \mathrm{meV}$ Pareto front are universally smaller than for those in the $0 \mathrm{meV}$ Pareto front. There is less of an impact on the FEL example, which could be due to the optima being highly sensitive to changes in the initial spot size.

Systems with insensitive optima (large $x$ ) will tolerate higher MTE photocathodes than the original characteristic MTE metric implies. Likewise, systems where the emittance grows rapidly for small changes in $\sigma_{x, i}(\operatorname{small} x$ ) cannot afford to decrease the initial spot size to compensate for any increase in the photocathode MTE. The second term in the square brackets of Eq. (5) is the relative scale for
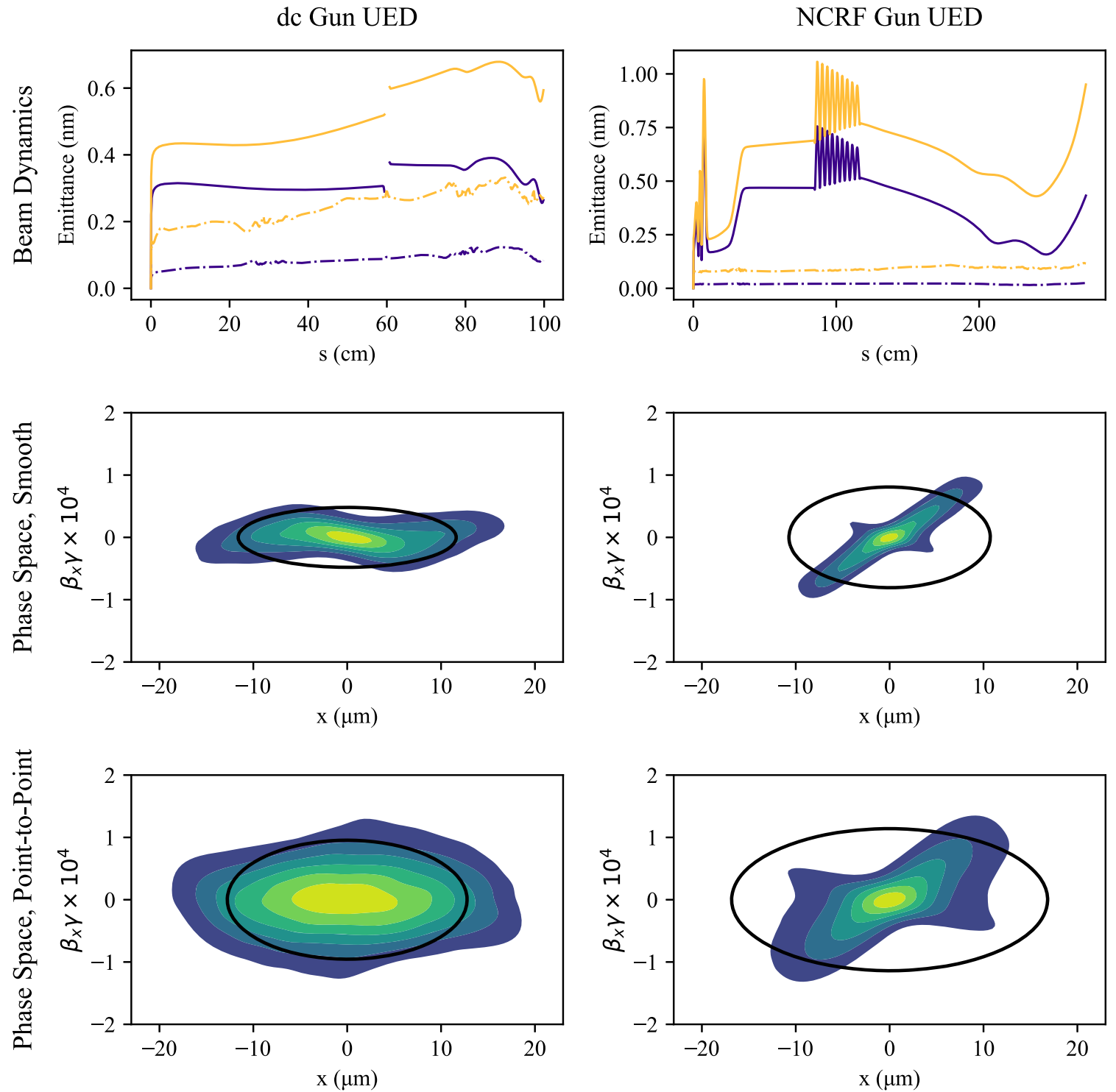

FIG. 8. The rms and core emittance of an individual with $10^{5}$ electrons per bunch from the dc gun UED and NCRF gun UED 0 meV MTE Pareto fronts. In the row labeled "beam dynamics", the yellow lines were computed with the point-to-point space-charge algorithm and the blue lines with smooth space charge. The solid lines are the rms normalized emittance, and the dashed lines are the core emittance. Below are plots of the beam's transverse phase space at the sample location computed with the smooth and point-to-point methods. Linear $x-p_{x}$ correlation has been removed, and the ellipse of phase space second moments is plotted in addition to the particle density. 
how much changing the initial spot size can improve emittance and can provide a rough guide to experimentalists for determining when a new photocathode technology requires reoptimization of the beam line. The MTE for which the transport and photocathode contributions to the final emittance are the same even when allowing the initial spot size to vary is

$$
\mathrm{MTE}_{C}^{\prime}=\operatorname{MTE}_{C}\left[1+\frac{x}{2}\right] \quad(x \ll 1) .
$$

Although analytical formulas for the optimal emittance and spot size which are accurate to all orders in $x$ may be found, they do not lend themselves to efficient analysis, and numerical methods may be better suited for investigating the properties of systems with insensitive optima.

For each system, we can use the Pareto fronts obtained for the 0 and $\sim 150 \mathrm{meV}$ MTE photocathodes to estimate the sensitivity parameter $x$ and calculate the correction to the characteristic MTE. These Pareto fronts give us a value of the optimal emittance from Eq. (5) for two different values of $\varepsilon_{\mathrm{C}}$, and from there we can solve for $x$. This operation was performed on each system, and the sensitivity parameter was used to calculate the corrected characteristic MTE. The correction in all cases was at the single percent level, indicating that our optima are sensitive to the initial spot size. Consequently, the uncorrected characteristic MTE, for the three realistic photoinjectors studied here, does a good job at predicting the scale at which photocathode improvements no longer improve brightness.

\section{STOCHASTIC SPACE CHARGE}

DIH is known to play a role in degrading the emittance of cold and dense electron beams. When the distance between particles falls below the Debye length of the onecomponent plasma, interparticle collisions can become important and can affect the momentum distribution of the bunch in a stochastic manner. This effect will show up prominently when the average kinetic energy of particles in the transverse direction is of the same scale as the potential energy due to the Coulomb repulsion of the particle's neighbors. The result is that the nascent momentum spread grows above its initial value by an amount $\Delta k T[\mathrm{eV}]=$ $1.04 \times 10^{-9}\left(n_{0}\left[\mathrm{~m}^{-3}\right]\right)^{1 / 3}[41,68]$. Using the electron number density $\left(n_{0}\right)$ at the beginning of each optimized example, the scale of DIH expected for all three beam lines is $1 \mathrm{meV}$. Beyond DIH, Coulomb scattering after the cathode can lead to continuous irreversible emittance growth, but these effects are difficult to estimate analytically. We expect DIH to be important in our simulations with the $0 \mathrm{meV}$ MTE photocathode due to the cold dense beams inside the guns.

To determine how much of an effect Coulomb scattering has on final emittance in our systems, one example from each of the dc and NCRF UED 0 meV Pareto fronts was chosen and simulated using a stochastic space-charge model. The new algorithm for efficiently computing the effects of stochastic space charge is based off of the BarnesHut tree method and will be discussed in detail in a forthcoming publication by Gordon, Maxson, et al. Both the NCRF and dc UED individuals had a bunch charge of 10 fC. Simulations were performed with GPT's smooth space-charge model discussed in Ref. [69] and with the tree-code method. The rms projected and core emittance [70] along each beam line and with each space-charge model are shown in Fig. 8. Coulomb scattering contributes a factor of 2 increase in final emittance for both cases.

\section{CONCLUSION}

We have shown that characteristic MTE can be a useful tool in understanding the scale of MTE at which photocathode improvements translate to an increase in usable brightness. These beam lines, which are representative of high-brightness photoinjector applications, have characteristic MTEs on the scale of single to tens of $\mathrm{meV}$, well below the $150 \mathrm{meV}$ MTE of today's commonly used photocathodes. Improvements in photocathode technology down to the level of $1 \mathrm{meV}$ and below stands to improve the brightness of practical photoinjectors by an impressive 2 orders of magnitude. However, it is not enough to simply insert a low-MTE photocathode into an electron gun to achieve low final emittance.

To achieve this level of photoinjector performance, advanced optimization techniques like MOGA will need to be integrated into the design and tuning of future accelerators. With the use of new photocathode technologies, further optimization may be required to take full advantage of low MTE. The sensitivity of the optima to changes in the initial spot size provides a guide for when it is necessary to reoptimize. In addition, when in the regime of single-meV photocathodes, existing models of smooth space charge break down, and the effects of Coulomb scattering become important in determining ultimate brightness. Although the results of the present work are not affected by this problem because we are concerned only with order of magnitude changes in emittance, design tools for future accelerators may need to move to highperformance point-to-point space-charge models to obtain good agreement with reality.

With the continued improvement of photocathode-based electron sources and, in particular, the reduction of MTE in photocathode materials, bright beams will open up new possibilities for accelerator physics applications. Notably, an increase in brightness would enable the time-resolved characterization of biological macromolecules with UED [71] as well as benefit X-ray FELs with a corresponding increase in total pulse energy benefiting a wide variety of $\mathrm{x}$-ray scattering experiments in fields ranging from condensed matter physics, to chemistry, to biology [72]. Work 
is already underway in understanding and beating the effects which limit photocathode MTE and in making existing low-MTE photocathodes more practical for accelerator facility use [73-75]. Additionally, structured particle emitters have already been predicted to mitigate the emittance growth observed from disorder-induced heating in the present simulations [76]. If these photocathode improvements can be realized, then their results could provide as much as 2 orders of magnitude improvement in the final brightness of realistic modern photoinjectors.

\section{ACKNOWLEDGMENTS}

This work was supported by the U.S. National Science Foundation under Grant No. PHY-1549132, the Center for Bright Beams. We thank the U.S.-Japan Science and Technology Cooperation Program in High Energy Physics for providing additional travel funding.

[1] B. J. Siwick, J. R. Dwyer, R. E. Jordan, and R. Miller, Femtosecond electron diffraction studies of strongly driven structural phase transitions, Chem. Phys. 299, 285 (2004).

[2] J. R. Dwyer, C. T. Hebeisen, R. Ernstorfer, M. Harb, V. B. Deyirmenjian, R. E. Jordan, and R. D. Miller, Femtosecond electron diffraction: 'making the molecu-lar movie', Phil. Trans. R. Soc. A 364, 741 (2006).

[3] C. W. Roberson, Y. Y. Lau, and H. P. Freund, Emittance, brightness, free-electron laser beam quality, and the scaled thermal velocity, in High-Brightness Accelerators, Vol. 178, edited by A. K. Hyder, M. F. Rose, and A. H. Guenther (Springer, Boston, 1988), pp. 627-645.

[4] S. Di Mitri and S. Spampinati, Estimate of free electron laser gain length in the presence of electron beam collective effects, Phys. Rev. Accel. Beams 17, 110702 (2014).

[5] I. V. Bazarov, B. M. Dunham, and C. K. Sinclair, Maximum Achievable Beam Brightness from Photoinjectors, Phys. Rev. Lett. 102, 104801 (2009).

[6] D. Filippetto, P. Musumeci, M. Zolotorev, and G. Stupakov, Maximum current density and beam brightness achievable by laser-driven electron sources, Phys. Rev. Accel. Beams 17, 024201 (2014).

[7] G. Shamuilov, A. Mak, K. Pepitone, and V. Goryashko, Child-Langmuir law for photoinjectors, Appl. Phys. Lett. 113, 204103 (2018).

[8] I. Pinayev et al., High-gradient High-charge CW Superconducting RF gun with $\mathrm{CsK} 2 \mathrm{Sb}$ photocathode, arXiv: 1511.05595 .

[9] B. M. Dunham, C. K. Sinclair, I. V. Bazarov, Y. Li, X. Liu, and K. W. Smolenski, Performance of a very high voltage photoemission electron gun for a high brightness, high average current ERL injector, in Proceedings of the 16th IEEE International Pulsed Power Conference, Albuquerque, NM (IEEE, Piscataway, NJ, 2007), pp. 1224-1226.

[10] D. H. Dowell, J. W. Lewellen, D. Nguyen, and R. Rimmer, The status of normal conducting RF (NCRF) guns, a summary of the ERL2005 workshop, Nucl. Instrum. Methods Phys. Res., Sect. A 557, 61 (2006).
[11] A. Arnold and J. Teichert, Overview on superconducting photoinjectors, Phys. Rev. Accel. Beams 14, 024801 (2011).

[12] M. Ferrario, J. E. Clendenin, D. T. Palmer, J. B. Rosenzweig, and L. Serafini, Homdyn study for the LCLS rf photoinjector, Technical Report No. SLAC-PUB 8400, INFN, Frascati, Italy, 2000.

[13] J. Rosenzweig, A. Cahill, B. Carlsten, G. Castorina, M. Croia, C. Emma, A. Fukusawa, B. Spataro, D. Alesini, V. Dolgashev, M. Ferrario, G. Lawler, R. Li, C. Limborg, J. Maxson, P. Musumeci, R. Pompili, S. Tantawi, and O. Williams, Ultra-high brightness electron beams from veryhigh field cryogenic radiofrequency photocathode sources, Nucl. Instrum. Methods Phys. Res., Sect. A 909, 224 (2018).

[14] A. Cahill, J. Rosenzweig, V. Dolgashev, S. Tantawi, and S. Weathersby, High gradient experiments with $\mathrm{X}$-band cryogenic copper accelerating cavities, Phys. Rev. Accel. Beams 21, 102002 (2018).

[15] X. J. Wang, M. Babzien, K. Batchelor, I. Ben-Zvi, R. I. Malone, X. Pogorelski, X. Qui, J. Sheehan, J. Sharitka, and T. Srinivasan-Rao, Experimental characterization of high-brightness electron photoinjector, in The Proceedings of FEL95, New York (JACoW Publishing, Geneva, Switzerland, 1995).

[16] J. Rosenzweig, A. Cahill, V. Dolgashev, C. Emma, A. Fukasawa, R. Li, C. Limborg, J. Maxson, P. Musumeci, A. Nause, R. Pakter, R. Pompili, R. Roussel, B. Spataro, and $\mathrm{S}$. Tantawi, Next generation high brightness electron beams from ultrahigh field cryogenic rf photocathode sources, Phys. Rev. Accel. Beams 22, 023403 (2019).

[17] A. H. McEuen, P. Lui, E. Tanabe, and V. Vaguine, , Highpower operation of accelerator structures at liquid nitrogen temperature, IEEE Trans. Nucl. Sci. 32, 2972 (1985).

[18] H. A. Schwettman, J. P. Turneaure, W. M. Fairbank, T. I. Smith, M. S. McAshan, P. B. Wilson, and E. E. Chambers, , Low temperature aspects of a cryogenic accelerator, IEEE Trans. Nucl. Sci. 14, 336 (1967).

[19] K. Nordlund and F. Djurabekova, Defect model for the dependence of breakdown rate on external electric fields, Phys. Rev. Accel. Beams 15, 071002 (2012).

[20] C. M. Fortgang, G. O. Bolme, J. Lamoureux, and D. J. Liska, Cryogenic experiments on rf accelerating structures, Nucl. Instrum. Methods Phys. Res., Sect. A 262, 197 (1987).

[21] A. Descoeudres, T. Ramsvik, S. Calatroni, M. Taborelli, and W. Wuensch, DC breakdown conditioning and breakdown rate of metals and metallic alloys under ultrahigh vacuum, Phys. Rev. Accel. Beams 12, 032001 (2009).

[22] A. Grudiev, S. Calatroni, and W. Wuensch, New local field quantity describing the high gradient limit of accelerating structures, Phys. Rev. Accel. Beams 12, 102001 (2009).

[23] V. Dolgashev, S. Tantawi, Y. Higashi, and B. Spataro, Geometric dependence of radio-frequency breakdown in normal conducting accelerating structures, Appl. Phys. Lett. 97, 171501 (2010).

[24] R. A. Marsh, M. A. Shapiro, R. J. Temkin, V. A. Dolgashev, L. L. Laurent, J. R. Lewandowski, A. D. Yeremian, and S. G. Tantawi, X-band photonic bandgap accelerator 
structure breakdown experiment, Phys. Rev. Accel. Beams 14, 021301 (2011).

[25] S. P. Weathersby et al., Mega-electron-volt ultrafast electron diffraction at SLAC National Accelerator Laboratory, Rev. Sci. Instrum. 86, 073702 (2015).

[26] J. Yang, K. Kan, N. Naruse, Y. Yoshida, K. Tanimura, and J. Urakawa, 100-femtosecond $\mathrm{MeV}$ electron source for ultrafast electron diffraction, Radiat. Phys. Chem. 78, 1106 (2009).

[27] Y. Ding, A. Brachmann, F.-J. Decker, D. Dowell, P. Emma, J. Frisch, S. Gilevich, G. Hays, P. Hering, Z. Huang, R. Iverson, H. Loos, A. Miahnahri, H.-D. Nuhn, D. Ratner, J. Turner, J. Welch, W. White, and J. Wu, Measurements and Simulations of Ultralow Emittance and Ultrashort Electron Beams in the Linac Coherent Light Source, Phys. Rev. Lett. 102, 254801 (2009).

[28] J. Maxson, D. Cesar, G. Calmasini, A. Ody, P. Musumeci, and D. Alesini, Direct Measurement of Sub-10 fs Relativistic Electron Beams with Ultralow Emittance, Phys. Rev. Lett. 118, 154802 (2017).

[29] S. Pastuszka, D. Kratzmann, D. Schwalm, A. Wolf, and A. S. Terekhov, Transverse energy spread of photoelectrons emitted from GaAs photocathodes with negative electron affinity, Appl. Phys. Lett. 71, 2967 (1997).

[30] L. Cultrera, S. Karkare, H. Lee, X. Liu, I. Bazarov, and B. Dunham, Cold electron beams from cryocooled, alkali antimonide photocathodes, Phys. Rev. Accel. Beams 18, 113401 (2015).

[31] P. Musumeci, J. G. Navarro, J. Rosenzweig, L. Cultrera, I. Bazarov, J. Maxson, S. Karkare, and H. Padmore, Advances in bright electron sources, Nucl. Instrum. Methods Phys. Res., Sect. A 907, 209 (2018).

[32] S. Karkare, A. Gowri, W. Andreas Schroeder, J. Kevin Nangoi, A. Tomas, M. Jared, and P. Howard, Ultracold Electrons via Near-Threshold Photoemission from SingleCrystal Cu(100), arXiv:2002.11579.

[33] B. Carlsten, New Photoelectric Injector Design for the Los Alamos National Laboratory XUV FEL Accelerator, in Proceedings of FEL88, Jerusalem, Israel (JACoW Publishing, Geneva, Switzerland, 1988).

[34] K. Floettmann, Emittance compensation in split photoinjectors, Phys. Rev. Accel. Beams 20, 013401 (2017).

[35] X. Qiu, K. Batchelor, I. Ben-Zvi, and X.-J. Wang, Demonstration of Emittance Compensation through the Measurement of the Slice Emittance of a 10-ps Electron Bunch, Phys. Rev. Lett. 76, 3723 (1996).

[36] L. Serafini and J. B. Rosenzweig, Envelope analysis of intense relativistic quasi laminar beams in rf photoinjectors: A theory of emittance compensation, Phys. Rev. E 55, 7565 (1997).

[37] B. Carlsten, Space-charge-induced emittance compensation in high-brightness photoinjectors, Part. Accel. 17, 27 (1995), https://cds.cern.ch/record/1108320/files/p27.pdf.

[38] I. V. Bazarov, A. Kim, M. N. Lakshmanan, and J. M. Maxson, Comparison of dc and superconducting rf photoemission guns for high brightness high average current beam production, Phys. Rev. Accel. Beams 14, 072001 (2011).
[39] O. A. Anderson, Internal dynamics and emittance growth in space-charge-dominated beams, Part. Accel. 21, 30 (1987), https://cds.cern.ch/record/1108052/files/p197.pdf.

[40] S. G. Anderson and J. B. Rosenzweig, Nonequilibrium Transverse motion and emittance growth in ultrarelativistic space-charge dominated beams, Phys. Rev. Accel. Beams 3, 094201 (2000).

[41] J. M. Maxson, I. V. Bazarov, W. Wan, H. A. Padmore, and C. E. Coleman-Smith, Fundamental photoemission brightness limit from disorder induced heating, New J. Phys. 15, 103024 (2013).

[42] K. Baptiste, J. Corlett, T. M. Huang, S. Kwiatkowski, D. Li, J. Qiang, F. Sannibale, J. Staples, R. Wells, L. Yang, A. Zholents, and J. McKenzie, Status of the LBNL NormalConducting CW VHF Photo-Injector, in Proceedings of the 23rd Particle Accelerator Conference, Vancouver, Canada, 2009 (IEEE, Piscataway, NJ, 2009), p. 4.

[43] E. Panofski, A. Jankowiak, T. Kamps, G. Kourkafas, and S. Eisebitt, Multi-objective Optimization of an SRF Photoinjector for ERL and UED Application, in Proceedings of IPAC2017, Copenhagen, Denmark (JACoW, Geneva, Switzerland, 2017), p. 4.

[44] Y. Ineichen, Massively parallel multi-objective optimization with application to particle accelerators, Ph.D. thesis, ETH Zurich, Switzerland, 2013.

[45] E. Panofski, A. Jankowiak, T. Kamps, and A. Neumann, Multi-objective optimization of an SRF photoinjectorwith booster section for high brightness beam performance, in Proceedings of the 9th International Particle Accelerator Conference, Vancouver, BC, Canada (JACOW Publishing, Geneva, Switzerland, 2018), p. 4.

[46] H. J. Qian, D. Filippetto, and F. Sannibale, S-band photoinjector investigations by multiobjective genetic optimizer, in Proceedings of IPAC2016, Busan, Korea (JACOW Publishing, Geneva, Switzerland, 2016).

[47] L. Emery, Global optimization of damping ring designs using a multi-objective evolutionary algorithm, in Proceedings of the 21st Particle Accelerator Conference, Knoxville, TN, 2005 (IEEE, Piscataway, NJ, 2005), pp. 2962-2964.

[48] C. F. Papadopoulos, J. N. Corlett, D. Filippetto, J. Qiang, F. Sannibale, J. W. Staples, M. Venturini, and M.S. Zolotorev, Multiobjective optimization for the advanced photoinjector experiment (APEX), in Proceedings of the 32nd Free Electron Laser Conference, Malmö, Sweden (Max-lab, Sweden, 2010), p. 4.

[49] K. Deb, A. Pratap, S. Agarwal, and T. Meyarivan, A fast and elitist multiobjective genetic algorithm: NSGA-II, IEEE Trans. Evol. Comput. 6, 182 (2002).

[50] G. Rudolph, Convergence of evolutionary algorithms in general search spaces, in Proceedings of IEEE International Conference on Evolutionary Computation, Nagoya, Japan (IEEE, New York, 1996), pp. 50-54, https://doi.org/10.1109/ICEC.1996.542332.

[51] I. V. Bazarov and C. K. Sinclair, Multivariate optimization of a high brightness dc gun photoinjector, Phys. Rev. Accel. Beams 8, 034202 (2005).

[52] S. van der Geer and M. de Loos, Applications of the General Particle Tracer code, in Proceedings of the Particle 
Accelerator Conference, Vancouver, BC, Canada, 1997 (IEEE, New York, 1997), Vol. 2, pp. 2577-2579.

[53] K. Floettmann, ASTRA: A Space Charge Tracking Algorithm (2017), http://www.desy.de/ mpyflo/Astra_ manual/Astra-Manual_V3.2.pdf.

[54] C. Gulliford, A. Bartnik, I. Bazarov, L. Cultrera, J. Dobbins, B. Dunham, F. Gonzalez, S. Karkare, H. Lee, H. Li, Y. Li, X. Liu, J. Maxson, C. Nguyen, K. Smolenski, and Z. Zhao, Demonstration of low emittance in the Cornell energy recovery linac injector prototype, Phys. Rev. Accel. Beams 16, 073401 (2013).

[55] K. Deb, Multi-Objective Optimization Using Evolutionary Algorithms (Wiley, New York, 2001), Vol. 16.

[56] E. Zitzler, M. Laumanns, and L. Thiele, SPEA2: Improving the strength Pareto evolutionary algorithm, Technical Report No. TIK-Report 103, ETH Library, Zurich, 2001.

[57] H. Lee, X. Liu, L. Cultrera, B. Dunham, V. O. Kostroun, and I. V. Bazarov, A cryogenically cooled high voltage DC photoemission electron source, Rev. Sci. Instrum. 89, 083303 (2018).

[58] C. Gulliford, A. Bartnik, and I. Bazarov, Multiobjective optimizations of a novel cryocooled dc gun based ultrafast electron diffraction beamline, Phys. Rev. Accel. Beams 19, 093402 (2016).

[59] M. Ligges, I. Rajkovic, P. Zhou, O. Posth, C. Hassel, G. Dumpich, and D. von der Linde, Observation Of ultrafast lattice heating using time resolved electron diffraction, Appl. Phys. Lett. 94, 101910 (2009).

[60] M. J. Stern, L. P. R. de Cotret, M. R. Otto, R. P. Chatelain, J.-P. Boisvert, M. Sutton, and B. J. Siwick, Mapping momentum-dependent electron-phonon coupling and nonequilibrium phonon dynamics with ultrafast electron diffuse scattering, Phys. Rev. B 97, 165416 (2018).

[61] P. Musumeci, M. S. Gutierrez, J. T. Moody, and C. M. Scoby, Time resolved relativistic electron diffraction, in Proceedings of the 23rd Particle Accelerator Conference, Vancouver, Canada, 2009 (IEEE, Piscataway, NJ, 2009), p. 3.

[62] P. Zhu, Y. Zhu, Y. Hidaka, L. Wu, J. Cao, H. Berger, J. Geck, R. Kraus, S. Pjerov, Y. Shen, R. I. Tobey, J. P. Hill, and X. J. Wang, Femtosecond time-resolved MeV electron diffraction, New J. Phys. 17, 063004 (2015).

[63] P. Zhu, J. Cao, Y. Zhu, J. Geck, Y. Hidaka, S. Pjerov, T. Ritschel, H. Berger, Y. Shen, R. Tobey, J. P. Hill, and X. J. Wang, Dynamic separation of electron excitation and lattice heating during the photoinduced melting of the periodic lattice distortion in $2 \mathrm{H}-\mathrm{TaSe} 2$, Appl. Phys. Lett. 103, 071914 (2013).

[64] D. Filippetto and H. Qian, Design of a high-flux instrument for ultrafast electron diffraction and microscopy, J. Phys. B 49, 104003 (2016).
[65] R. B. Neal, The Stanford two mile accelerator, Technical Report No. SLAC-REPRINT-1968-001, Stanford Linear Accelerator Center, Menlo Park, CA, 1968.

[66] C. Gulliford, A. Bartnik, I. Bazarov, and J. Maxson, Multiobjective optimization design of an rf gun based electron diffraction beam line, Phys. Rev. Accel. Beams 20, 033401 (2017).

[67] T. Konomi, Y. Honda, E. Kako, Y. Kobayashi, S. Michizono, T. Miyajima, H. Sakai, K. Umemori, and S. Yamaguchi, Development of High Intensity, High Brightness, CW SRF Gun with Bi-Alkali Photocathode, Dresden, Germany (JACoW, Geneva, Switzerland, 2019), p. 4.

[68] S. B. van der Geer, M. P. Reijnders, M. J. de Loos, E. J. D. Vredenbregt, P. H. A. Mutsaers, and O. J. Luiten, Simulated performance of an ultracold ion source, J. Appl. Phys. 102, 094312 (2007).

[69] G. Poplau, U. van Rienen, M. de Loos, and B. van der Geer, Fast calculation of space charge in beam line tracking by multigrid techniques, in Scientific Computing in Electrical Engineering, Vol. 4, edited by H.-G. Bock, F. d. Hoog, A. Friedman, A. Gupta, W. Langford, H. Neunzert, W. R. Pulleyblank, T. Rusten, F. Santosa, A.-K. Tornberg, W. H. A. Schilders, E. J. W. ter Maten, and S. H. M. J. Houben (Springer, Berlin, 2004), pp. 329-336.

[70] I. V. Bazarov, Synchrotron radiation representation in phase space, Phys. Rev. Accel. Beams 15, 050703 (2012).

[71] G. Sciaini and R. J.D. Miller, Femtosecond electron diffraction: heralding the era of atomically resolved dynamics, Rep. Prog. Phys. 74, 096101 (2011).

[72] P. Abbamonte, F. Abild-Pederse, P. Adams, M. Ahmed, and F. Albert, New science opportunities enabled by LCLS-II x-r lasers, Technical Report No. SLAC-R-1053, SLAC, Menlo Park, CA, 2015.

[73] S. Karkare and I. Bazarov, Effect of nano-scale surface roughness on transverse energy spread from GaAs photocathodes, Appl. Phys. Lett. 98, 094104 (2011).

[74] L. Jones, D. Juarez-Lopez, B. Militsyn, T. Noakes, and C. Welsch, Transverse energy distribution measurements for polycrystalline and (100) copper photocathodes with known levels of surface roughness, in Proceedings of the 9th International Particle Accelerator Conference, IPAC2018, Vancouver, British Columbia, Canada (JACOW Publishing, Geneva, Switzerland, 2018), https:// doi.org/10.18429/jacow-ipac2018-thpmk062.

[75] J. Feng, J. Nasiatka, W. Wan, S. Karkare, J. Smedley, and H. A. Padmore, Thermal limit to the intrinsic remittance from metal photocathodes, Appl. Phys. Lett. 107, 134101 (2015).

[76] D. Murphy, R. Scholten, and B. Sparkes, Increasing the Brightness of Cold Ion Beams by Suppressing DisorderInduced Heating with Rydberg Blockade, Phys. Rev. Lett. 115, 214802 (2015). 